\title{
Antigen-directed therapies: an effective tool in acute myeloid leukemia?
}

\author{
"With many antigen-specific therapies currently under \\ development for acute myeloid leukemia, there should be \\ optimism that we can soon expand the array of treatment \\ options for our patients."
}

First draft submitted: 6 June 2016; Accepted for publication: 22 June 2016; Published online: 8 September 2016

Keywords: acute myeloid leukemia $\bullet$ antibody $\bullet$ antigen $\bullet$ CD33 $\bullet$ gemtuzumab ozogamicin - immunotherapy

Outcomes with intensive chemotherapy and hematopoietic cell transplantation have gradually improved over time in acute myeloid leukemia (AML), but therapeutic failure remains common, and most patients still die from consequences of persistent or recurrent disease or from treatment-related toxicities [1]. Interest in antigen-directed therapies as a means of overcoming these inadequacies had first arisen over 40 years ago [2] and has been sustained ever since. Success so far has been modest [3]. Not for the lack of trying: efforts have spanned multiple target antigens and involved diverse classes of therapeutics including unconjugated antibodies, antibodies armed with anticancer effectors (toxins, small molecule drugs or radionuclides) and vaccines. A large number of agents have shown antileukemic properties in preclinical studies and have subsequently entered the clinic. In some cases, investigations are ongoing, and the drug's value has yet to be defined. In many cases, however, the development has been abandoned based on disappointing patient results. Testing was often short-lived and did not go beyond uncontrolled early phase trials, but two unconjugated antibodies - one targeting CD33 (lintuzumab, also known as SGN-33 or HuM195) and one targeting VEGF (bevacizumab) - failed only at the stage of randomized trials [4-6]. Thus, rather than proving effective, most agents tested to date have highlighted the challenges of using antigen-directed therapies in AML.
Nonetheless, one of the only new AML drugs to improve survival in randomized trials in the last three decades is the CD33 antibody-drug conjugate (ADC) gemtuzumab ozogamicin (GO) [7-9]. The clinical experience with GO unequivocally demonstrates that antigen-directed therapies can be effective for a disease as diverse as AML as long as suitable patient subsets are identified. In light of the many immunotherapeutics that have failed, this success is particularly remarkable since GO is by no means a perfect drug. Besides concerns over the stability of the linker, the conjugation technology of this first-generation ADC was not yet optimized, and roughly half of the antibody molecules remained unlabeled. Perhaps more significantly, the calicheamicin derivative utilized as toxic payload in $\mathrm{GO}$ - while highly potent - is an excellent substrate for drug transporter proteins, in particular P-glycoprotein [10]. These transporters have long been recognized as an important resistance mechanism for AML cells [11] and likely contribute to the cross-resistance observed between GO and conventional AML chemotherapeutics.

Correlative analyses from randomized trials have also clarified cellular determinants for the clinical activity of GO, including target antigen expression levels on leukemic blasts and, at least in adults, cytogenetic and molecular characteristics [7,12,13]. Although it is not entirely surprising that GO is more effective in certain patients, while it is less

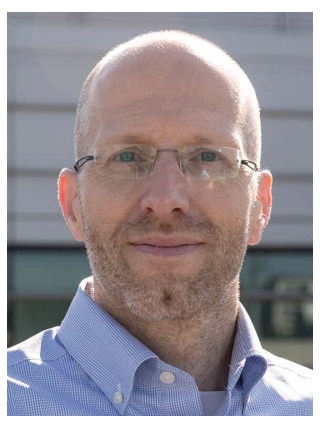

Roland B Walter ${ }^{*}, 1,2,3$ ${ }^{1}$ Clinical Research Division, Fred Hutchinson Cancer Research Center, Seattle, WA, USA

2Department of Medicine, Division of Hematology, University of Washington, Seattle, WA, USA

${ }^{3}$ Department of Epidemiology, University of Washington, Seattle, WA, USA *Author for correspondence:

Tel.: +1 2066673599

Fax: +1 2066676519 rwalter@fredhutch.org 
useful or even ineffective or hurtful in others, such interindividual differences have largely been ignored throughout much of the drug's clinical development. To some degree, this may have occurred inadvertently. For instance, the restriction to patients in first relapse with a first remission duration of 3 months or longer in the early Phase II monotherapy trials [14] may have been useful to 'homogenize' the study population and simplify data interpretation, but may have led to an underappreciation of the impact of genetic or molecular disease features. Still, there were early hints, for example from $\mathrm{X}$ chromosome inactivation studies, pointing at differences in the stem and progenitor cell composition between individual patients [15]. These analyses directly suggested that long-term benefit from CD33-targeted therapy may be limited to distinct patient subsets. The early recognition of the association between P-glycoprotein and clinical resistance to GO [16] was another early clue that some patients would be better candidates for GO therapy than others. In retrospect, one could argue that the conduct of clinical trials with designs that reflect the believe that GO would be a drug that fits all - rather than considering the biologic diversity of AML - is a 'self-inflicted' reason why not only did it take many years following accelerated marketing approval by the US FDA before the value of GO became apparent, but also why this drug is currently no longer available in most commercial markets around the globe. Specifically, the confirmatory Phase III trial (SWOG S0106), mandated under FDA's Accelerated Approval regulations, was designed - and failed - to demonstrate a survival benefit with GO across all cytogenetic risk groups [17]. It is conceivable that earlier attention to response biomarkers would have led to an alternative design of the pivotal Phase III trial and, plausibly, the confirmation of patient benefit. It can only be hoped that GO finds its way back into the armamentarium of AML therapeutics. At the minimum, however, we need to use insight from the lessons learned during the development of this ADC as they inform on principles that should be pertinent to the evaluation of other antigen-directed therapies and may help avoid mistakes during the testing of new agents that have far-reaching consequences. GO's fate is a solemn reminder of how shortcomings during clinical development can deprive patients from active drugs.

Efficient drug testing has never been as important as today, with early clinical findings suggesting antiAML activity of several unconjugated and conjugated antibodies and, perhaps, vaccines and adoptive immunotherapies with cells expressing modified T-cell receptors [3]. In patients with acute lymphoblastic leukemia and other lymphoid malignancies, impressive activity has been observed with new classes of immunotherapeutics, most notably small bispecific antibodies and chimeric antigen receptor-modified immune effector cells [18]. First-in-human trials of such therapies for AML have been initiated over the last year and others will soon follow. This exploration of a wide range of therapeutics is paralleled by efforts to target an expanding variety of antigens on, in or around AML cells [3] - a reflection of the antigenic heterogeneity that constitutes a considerable hurdle for all antigen-directed therapeutics. In a disease as diverse as AML, there may be no single optimal antigen, and for any particular antigen, only a subset of patients may be suitable. Maximal treatment benefit may even require targeting of more than one antigen, in addition to integration of conventional chemotherapeutics or immune-stimulating drugs. To complicate matters further, the choice of target antigen(s) - or sequence of use - may depend on the main therapy purpose (e.g., gross reduction of tumor burden vs eradication of small amounts of residual leukemia vs deletion of the very rare underlying malignant stem cells). With an increasing number of experimental therapeutics at our disposal, timely identification of the critical determinants for the antileukemic activity of each of them will be critical to optimize their use for patients and facilitate an eventual path to regulatory approval. While perhaps perceived as 'lowhanging fruit', centering drug-development strategies on relatively unselected patient populations, such as older adults with medical comorbidities and intolerance to intensive conventional chemotherapies, may prove less than ideal to unravel a targeted drug's true benefit.

$\mathrm{GO}$ also serves as a reminder that the intricate balance between efficacy and toxicity applies to antigendirected therapies as well $[19,20]$. This is particularly important now that we enter an era of highly potent therapeutics. Life-threatening toxicities with novel immunotherapies such as small bispecific antibodies and gene-modified immune effector cells are well recognized in other cancers [18]. While the toxicity profiles of similar drugs have yet to be established for AML, substantial side effects have to be anticipated, as none of the currently pursued antigens are leukemia specific. All are found on normal mature and/or immature cells, particularly within the hematopoietic system. More potent drugs may adversely affect a larger array of normal cells as they may hit hematopoietic cells that were protected from less potent drugs through low target antigen expression, drug transporter proteins or other mechanisms. More extensive 'on-target, off AML' effects may then cause longer and more severe cytopenias and put patients at higher risk for life-threatening complications, especially bleeding and infections. 
Therefore, it remains to be tested whether more potent drugs will indeed provide a useful therapeutic window and greater benefit than less potent agents. They may not, and it is conceivable that some highly potent therapeutics targeting CD33 or other AML-associated antigens may require autologous or allogeneic stem cell rescue for safe administration to abrogate their effects on normal hematopoietic cells and/or immune effector cells - therapies with their own risks that may curtail or nullify any benefit of the novel therapeutic. With differing requirements for support care, the ideal format of immunotherapy may not only depend on target antigen but also exact treatment situation, for example, disease stage and patient characteristics.

Undoubtedly, we are entering an exciting time. With many antigen-specific therapies currently under development for AML, there should be optimism that we can soon expand the array of treatment options for our patients. GO was a good start - it not only yielded a strong glimpse of the potential value of

\section{References}

1 Döhner H, Weisdorf DJ, Bloomfield CD. Acute myeloid leukemia. N. Engl. J. Med. 373(12), 1136-1152 (2015).

2 Harris R. Leukaemia antigens and immunity in man. Nature 241(5385), 95-100 (1973).

3 Buckley SA, Walter RB. Antigen-specific immunotherapies for acute myeloid leukemia. Hematol. Am. Soc. Hematol. Educ. Program 2015(1), 584-595 (2015).

4 Feldman EJ, Brandwein J, Stone R et al. Phase III randomized multicenter study of a humanized anti-CD33 monoclonal antibody, lintuzumab, in combination with chemotherapy, versus chemotherapy alone in patients with refractory or first-relapsed acute myeloid leukemia. J. Clin. Oncol. 23(18), 4110-4116 (2005).

5 Sekeres MA, Lancet JE, Wood BL et al. Randomized Phase IIb study of low-dose cytarabine and lintuzumab versus lowdose cytarabine and placebo in older adults with untreated acute myeloid leukemia. Haematologica 98(1), 119-128 (2013).

6 Ossenkoppele GJ, Stussi G, Maertens J et al. Addition of bevacizumab to chemotherapy in acute myeloid leukemia at older age: a randomized Phase 2 trial of the Dutch-Belgian Cooperative Trial Group for Hemato-Oncology (HOVON) and the Swiss Group for Clinical Cancer Research (SAKK). Blood 120(24), 4706-4711 (2012).

7 Hills RK, Castaigne S, Appelbaum FR et al. Addition of gemtuzumab ozogamicin to induction chemotherapy in adult patients with acute myeloid leukaemia: a meta-analysis of individual patient data from randomised controlled trials. Lancet Oncol. 15(9), 986-996 (2014).

8 Gamis AS, Alonzo TA, Meshinchi S et al. Gemtuzumab ozogamicin in children and adolescents with de novo acute myeloid leukemia improves event-free survival by reducing relapse risk: results from the randomized Phase III Children's antigen-directed therapies but also provided important insight into the do's and don'ts of testing of such drugs that are likely pertinent to the evaluation of the new generation of AML immunotherapeutics as well.

\section{Financial \& competing interests disclosure}

The author is a Leukemia \& Lymphoma Society Scholar in Clinical Research. The author has received commercial research grants from Amgen Inc., Amphivena Therapeutics, Inc., Covagen AG and Seattle Genetics, Inc.; has ownership interests with Amphivena Therapeutics, Inc. and has been a consultant to Agios Pharmaceuticals, Inc., Amphivena Therapeutics, Inc., Covagen AG, Emergent Biosolutions, Inc., Janssen Research \& Development, LLC, Pfizer, Inc. and Seattle Genetics, Inc. The author has no other relevant affiliations or financial involvement with any organization or entity with a financial interest in or financial conflict with the subject matter or materials discussed in the manuscript apart from those disclosed.

No writing assistance was utilized in the production of this manuscript.

Oncology Group trial AAML0531. J. Clin. Oncol. 32(27), 3021-3032 (2014).

9 Amadori S, Suciu S, Selleslag D et al. Gemtuzumab ozogamicin versus best supportive care in older patients with newly diagnosed acute myeloid leukemia unsuitable for intensive chemotherapy: results of the randomized Phase III EORTC-GIMEMA AML-19 trial. J. Clin. Oncol. 34(9), 972-979 (2016).

10 Laszlo GS, Estey EH, Walter RB. The past and future of $\mathrm{CD} 33$ as therapeutic target in acute myeloid leukemia. Blood Rev. 28(4), 143-153 (2014).

11 De Jonge-Peeters SD, Kuipers F, De Vries EG, Vellenga E. $\mathrm{ABC}$ transporter expression in hematopoietic stem cells and the role in AML drug resistance. Crit. Rev. Oncol. Hematol. 62(3), 214-226 (2007).

12 Pollard JA, Loken M, Gerbing RB et al. CD33 expression and its association with gemtuzumab ozogamicin response: results from the randomized Phase III Children's Oncology Group trial AAML0531. J. Clin. Oncol. 34(7), 747-755 (2016).

13 Olombel G, Guerin E, Guy J et al. The level of blast CD33 expression positively impacts the effect of gemtuzumab ozogamicin in patients with acute myeloid leukemia. Blood 127(17), 2157-2160 (2016).

14 Larson RA, Sievers EL, Stadtmauer EA et al. Final report of the efficacy and safety of gemtuzumab ozogamicin (Mylotarg) in patients with CD33-positive acute myeloid leukemia in first recurrence. Cancer 104(7), 1442-1452 (2005).

15 Walter RB, Appelbaum FR, Estey EH, Bernstein ID. Acute myeloid leukemia stem cells and CD33-targeted immunotherapy. Blood 119(26), 6198-6208 (2012).

16 Linenberger ML, Hong T, Flowers D et al. Multidrugresistance phenotype and clinical responses to gemtuzumab 
ozogamicin. Blood 98(4), 988-994 (2001).

17 Petersdorf SH, Kopecky KJ, Slovak M et al. A Phase III study of gemtuzumab ozogamicin during induction and postconsolidation therapy in younger patients with acute myeloid leukemia. Blood 121(24), 4854-4860 (2013).

18 Batlevi CL, Matsuki E, Brentjens RJ, Younes A. Novel immunotherapies in lymphoid malignancies. Nat. Rev. Clin. Oncol. 13(1), 25-40 (2016).

19 Amadori S, Suciu S, Stasi R et al. Sequential combination of gemtuzumab ozogamicin and standard chemotherapy in older patients with newly diagnosed acute myeloid leukemia: results of a randomized Phase III trial by the EORTC and GIMEMA consortium (AML-17). J. Clin. Oncol. 31(35), 4424-4430 (2013).

20 Burnett A, Cavenagh J, Russell N et al. Defining the dose of gemtuzumab ozogamicin in combination with induction chemotherapy in acute myeloid leukemia: a comparison of $3 \mathrm{mg} / \mathrm{m}^{2}$ with $6 \mathrm{mg} / \mathrm{m}^{2}$ in the NCRI AML17 Trial. Haematologica 101(6), 724-731 (2016). 